EUROPEAN ORGANIZATION FOR NUCLEAR RESEARCH

EUROPEAN LABORATORY FOR PARTICLE PHYSICS (CERN)

CERN-PPE/92-210

January 13,1993

\title{
Search for Particles with Unexpected Mass and Charge in $\mathrm{Z}$ Decays
}

The ALEPH Collaboration

\begin{abstract}
During 1989 and 1990 over 180000 hadronic and leptonic events, corresponding to $8 \mathrm{pb}^{-1}$ of luminosity, were collected by the ALEPH detector in a scan of the Z peak at the $e^{+} e^{-}$collider LEP. This letter reports the results of a search in these data for particles with unexpected mass and charge by measurement of the ionization energy loss of charged tracks in the ALEPH TPC central tracking detector. The mass limits for the pair production of fractionally charged particles and of heavy, long lived charged particles are extended to $43 \mathrm{GeV} / c^{2}$ at $90 \%$ confidence level. If single production of a heavy particle is considered, the mass limit is extended to more than $70 \mathrm{GeV} / c^{2}$.
\end{abstract}

Submitted to Physics Letters B 


\section{The ALEPH Collaboration}

D. Buskulic, D. Decamp, C. Goy, J.-P. Lees, M.-N. Minard, B. Mours

Laboratoire de Physique des Particules (LAPP), IN P $^{3}$-CNRS, 74019 Annecy-le-Vieux Cedex, France

R. Alemany, F. Ariztizabal, P. Comas, J.M. Crespo, M. Delfino, E. Fernandez, V. Gaitan, Ll. Garrido, T. Mattison,

A. Pacheco, C. Padilla, A. Pascual Institut de Fisica d'Altes Energies, Universitat Autonoma de Barcelona, 08193 Bellaterra (Barcelona), $\operatorname{Spain}^{7}$

D. Creanza, M. de Palma, A. Farilla, G. Iaselli, G. Maggi, M. Maggi, S. Natali, S. Nuzzo, M. Quattromini,

A. Ranieri, G. Raso, F. Romano, F. Ruggieri, G. Selvaggi, L. Silvestris, P. Tempesta, G. Zito

INFN Sezione di Bari e Dipartimento di Fisica dell' Università, 70126 Bari, Italy

H. Hu, ${ }^{20}$ D. Huang, X. Huang, J. Lin, J. Lou, C. Qiao, ${ }^{20}$ T. Wang, Y. Xie, D. Xu, R. Xu, J. Zhang, W. Zhao Institute of High-Energy Physics, Academia Sinica, Beijing, The People's Republic of China ${ }^{8}$

L.A.T. Bauerdick, ${ }^{24}$ E. Blucher, G. Bonvicini, J. Boudreau, D. Casper, H. Drevermann, R.W. Forty, G. Ganis, C. Gay, R. Hagelberg, J. Harvey, S. Haywood, J. Hilgart, R. Jacobsen, B. Jost, J. Knobloch, E. Lançon, I. Lehraus, T. Lohse, ${ }^{32}$ A. Lusiani, M. Martinez, P. Mato, H. Meinhard, A. Minten, R. Miquel, H.-G. Moser, P. Palazzi, J.A. Perlas, J.-F. Pusztaszeri, ${ }^{30}$ F. Ranjard, G. Redlinger, ${ }^{25}$ L. Rolandi, J. Rothberg, ${ }^{2}$ T. Ruan, ${ }^{20,27}$ M. Saich, D. Schlatter, M. Schmelling, F. Sefkow, W. Tejessy, H. Wachsmuth, W. Wiedenmann, T. Wildish, W. Witzeling, J. Wotschack

European Laboratory for Particle Physics (CERN), 1211 Geneva 23, Switzerland

Z. Ajaltouni, F. Badaud, M. Bardadin-Otwinowska, A.M. Bencheikh, R. El Fellous, A. Falvard, P. Gay,

C. Guicheney, P. Henrard, J. Jousset, B. Michel, J-C. Montret, D. Pallin, P. Perret, B. Pietrzyk, J. Proriol, F. Prulhière, G. Stimpfl

Laboratoire de Physique Corpusculaire, Université Blaise Pascal, IN ${ }^{2} P^{3}$-CNRS, Clermont-Ferrand, 63177 Aubière, France

T. Fearnley, J.D. Hansen, J.R. Hansen, ${ }^{1}$ P.H. Hansen, R. Møllerud, B.S. Nilsson

Niels Bohr Institute, 2100 Copenhagen, Denmark ${ }^{9}$

I. Efthymiopoulos, A. Kyriakis, E. Simopoulou, A. Vayaki, K. Zachariadou

Nuclear Research Center Demokritos (NRCD), Athens, Greece

J. Badier, A. Blondel, G. Bonneaud, J.C. Brient, G. Fouque, S. Orteu, A. Rougé, M. Rumpf, R. Tanaka, M. Verderi, H. Videau

Laboratoire de Physique Nucléaire et des Hautes Energies, Ecole Polytechnique, IN P $^{3}$-CNRS, 91128 Palaiseau Cedex, France

D.J. Candlin, M.I. Parsons, E. Veitch

Department of Physics, University of Edinburgh, Edinburgh EH9 3JZ, United Kingdom ${ }^{10}$

L. Moneta, G. Parrini

Dipartimento di Fisica, Università di Firenze, INFN Sezione di Firenze, 50125 Firenze, Italy

M. Corden, C. Georgiopoulos, M. Ikeda, J. Lannutti, D. Levinthal, ${ }^{15}$ M. Mermikides ${ }^{\dagger}$, L. Sawyer, S. Wasserbaech Supercomputer Computations Research Institute and Dept. of Physics, Florida State University, Tallahassee, FL 32306, USA 12,13,14

A. Antonelli, R. Baldini, G. Bencivenni, G. Bologna, ${ }^{4}$ F. Bossi, P. Campana, G. Capon, F. Cerutti, V. Chiarella, B. D'Ettorre-Piazzoli, ${ }^{26}$ G. Felici, P. Laurelli, G. Mannocchi, ${ }^{5}$ F. Murtas, G.P. Murtas, L. Passalacqua, M. PepeAltarelli, P. Picchi ${ }^{4}$

Laboratori Nazionali dell'INFN (LNF-INFN), 00044 Frascati, Italy 
P. Colrain, I. ten Have, J.G. Lynch, W. Maitland, W.T. Morton, C. Raine, J.M. Scarr, K. Smith, M.G. Smith, A.S. Thompson, R.M. Turnbull

Department of Physics and Astronomy, University of Glasgow, Glasgow G12 8QQ, United Kingdom ${ }^{10}$

B. Brandl, O. Braun, C. Geweniger, P. Hanke, V. Hepp, E.E. Kluge, Y. Maumary, A. Putzer, B. Rensch, A. Stahl, K. Tittel, M. Wunsch

Institut für Hochenergiephysik, Universität Heidelberg, 6900 Heidelberg, Fed. Rep. of Germany ${ }^{16}$

A.T. Belk, R. Beuselinck, D.M. Binnie, W. Cameron, M. Cattaneo, D.J. Colling, P.J. Dornan, S. Dugeay, A.M. Greene, J.F. Hassard, N.M. Lieske, J. Nash, S.J. Patton, D.G. Payne, M.J. Phillips, J.K. Sedgbeer, I.R. Tomalin, A.G. Wright

Department of Physics, Imperial College, London SW7 2BZ, United Kingdom ${ }^{10}$

E. Kneringer, D. Kuhn, G. Rudolph

Institut für Experimentalphysik, Universität Innsbruck, 6020 Innsbruck, Austria $^{18}$

C.K. Bowdery, T.J. Brodbeck, A.J. Finch, F. Foster, G. Hughes, D. Jackson, N.R. Keemer, M. Nuttall, A. Patel, T. Sloan, S.W. Snow, E.P. Whelan

Department of Physics, University of Lancaster, Lancaster LA1 4YB, United Kingdom ${ }^{10}$

K. Kleinknecht, J. Raab, B. Renk, H.-G. Sander, H. Schmidt, F. Steeg, S.M. Walther, R. Wanke, B. Wolf Institut für Physik, Universität Mainz, 6500 Mainz, Fed. Rep. of Germany ${ }^{16}$

J-J. Aubert, C. Benchouk, A. Bonissent, J. Carr, P. Coyle, J. Drinkard, F. Etienne, S. Papalexiou, P. Payre, Z. Qian, L. Roos, D. Rousseau, P. Schwemling, M. Talby

Centre de Physique des Particules, Faculté des Sciences de Luminy, IN ${ }^{2} P^{3}$-CNRS, 13288 Marseille, France

S. Adlung, R. Assmann, C. Bauer, W. Blum, ${ }^{1}$ D. Brown, P. Cattaneo, ${ }^{31}$ B. Dehning, H. Dietl, F. Dydak, ${ }^{23}$ M. Fernandez-Bosman, M. Frank, A.W. Halley, J. Lauber, G. Lütjens, G. Lutz, W. Männer, R. Richter,

H. Rotscheidt, J. Schröder, A.S. Schwarz, R. Settles, H. Seywerd, U. Stierlin, U. Stiegler, R. St. Denis,

M. Takashima, ${ }^{3}$ J. Thomas, ${ }^{3}$ G. Wolf Max-Planck-Institut für Physik, Werner-Heisenberg-Institut, 8000 München, Fed. Rep. of Germany ${ }^{16}$

J. Boucrot, O. Callot, A. Cordier, M. Davier, L. Duflot, J.-F. Grivaz, Ph. Heusse, D.E. Jaffe, P. Janot, D.W. Kim, 19

F. Le Diberder, J. Lefrançois, A.-M. Lutz, M.-H. Schune, J.-J. Veillet, I. Videau, Z. Zhang, Laboratoire de l'Accélérateur Linéaire, Université de Paris-Sud, IN ${ }^{2} P^{3}$ CNRS, 91405 Orsay Cedex, France

D. Abbaneo, S.R. Amendolia, G. Bagliesi, G. Batignani, L. Bosisio, U. Bottigli, C. Bozzi, C. Bradaschia, M. Carpinelli, M.A. Ciocci, R. Dell'Orso, I. Ferrante, F. Fidecaro, L. Foà, E. Focardi, F. Forti, A. Giassi, M.A. Giorgi, F. Ligabue, E.B. Mannelli, P.S. Marrocchesi, A. Messineo, F. Palla, G. Rizzo, G. Sanguinetti, P. Spagnolo, J. Steinberger, R. Tenchini, G. Tonelli, G. Triggiani, C. Vannini, A. Venturi, P.G. Verdini, J. Walsh Dipartimento di Fisica dell'Università, INFN Sezione di Pisa, e Scuola Normale Superiore, 56010 Pisa, Italy

A.P. Betteridge, J.M. Carter, M.G. Green, P.V. March, Ll.M. Mir, T. Medcalf, I.S. Quazi, J.A. Strong, L.R. West Department of Physics, Royal Holloway \& Bedford New College, University of London, Surrey TW20 OEX, United Kingdom ${ }^{10}$

D.R. Botterill, R.W. Clifft, T.R. Edgecock, M. Edwards, S.M. Fisher, T.J. Jones, P.R. Norton, D.P. Salmon, J.C. Thompson

Particle Physics Dept., Rutherford Appleton Laboratory, Chilton, Didcot, Oxon OX11 OQX, United King dom ${ }^{10}$ 
B. Bloch-Devaux, P. Colas, H. Duarte, W. Kozanecki, M.C. Lemaire, E. Locci, E. Monnier, P. Perez, F. Perrier, J. Rander, J.-F. Renardy, A. Rosowsky, A. Roussarie, J.-P. Schuller, J. Schwindling, D. Si Mohand, B. Vallage Service de Physique des Particules, DAPNIA, CE-Saclay, 91191 Gif-sur-Yvette Cedex, France ${ }^{17}$

R.P. Johnson, A.M. Litke, G. Taylor, J. Wear Institute for Particle Physics, University of California at Santa Cruz, Santa Cruz, CA 95064, USA ${ }^{29}$

J.G. Ashman, W. Babbage, C.N. Booth, C. Buttar, R.E. Carney, S. Cartwright, F. Combley, F. Hatfield, P. Reeves, L.F. Thompson ${ }^{1}$

Department of Physics, University of Sheffield, Sheffield S3 7RH, United Kingdom ${ }^{10}$

E. Barberio, A. Böhrer, S. Brandt, G. Cowan, C. Grupen, G. Lutters, F. Rivera, U. Schäfer Fachbereich Physik, Universität Siegen, 5900 Siegen, Fed. Rep. of Germany ${ }^{16}$

R. Della Marina, G. Giannini, B. Gobbo, F. Ragusa ${ }^{22}$ Dipartimento di Fisica, Università di Trieste e INFN Sezione di Trieste, 34127 Trieste, Italy

L. Bellantoni, W. Chen, D. Cinabro, ${ }^{28}$ J.S. Conway, D.F. Cowen, ${ }^{21}$ Z. Feng, D.P.S. Ferguson, Y.S. Gao, J. Grahl, J.L. Harton, R.C. Jared, ${ }^{6}$ B.W. LeClaire, C. Lishka, A. Miotto, Y.B. Pan, J.R. Pater, Y. Saadi, V. Sharma, M. Schmitt, Z.H. Shi, A.M. Walsh, F.V. Weber, M.H. Whitney, Sau Lan Wu, X. Wu, G. Zobernig Department of Physics, University of Wisconsin, Madison, WI 53706, USA ${ }^{11}$

\footnotetext{
$\dot{\phi}$ Deceased.

${ }^{1}$ Now at CERN, PPE Division, 1211 Geneva 23, Switzerland.

${ }^{2}$ Permanent address: University of Washington, Seattle, WA 98195, USA.

${ }^{3}$ Now at SSCL, Dallas, TX, U.S.A.

${ }^{4}$ Also Istituto di Fisica Generale, Università di Torino, Torino, Italy.

${ }^{5}$ Also Istituto di Cosmo-Geofisica del C.N.R., Torino, Italy.

${ }^{6}$ Permanent address: LBL, Berkeley, CA 94720, USA.

${ }^{7}$ Supported by CICYT, Spain.

${ }^{8}$ Supported by the National Science Foundation of China.

${ }^{9}$ Supported by the Danish Natural Science Research Council.

${ }^{10}$ Supported by the UK Science and Engineering Research Council.

${ }^{11}$ Supported by the US Department of Energy, contract DE-AC02-76ER00881.

${ }^{12}$ Supported by the US Department of Energy, contract DE-FG05-87ER40319.

${ }^{13}$ Supported by the NSF, contract PHY-8451274.

${ }^{14}$ Supported by the US Department of Energy, contract DE-FC0S-85ER250000.

${ }^{15}$ Supported by SLOAN fellowship, contract BR 2703.

${ }^{16}$ Supported by the Bundesministerium für Forschung und Technologie, Fed. Rep. of Germany.

${ }^{17}$ Supported by the Direction des Sciences de la Matière, C.E.A.

${ }^{18}$ Supported by Fonds zur Förderung der wissenschaftlichen Forschung, Austria.

${ }^{19}$ Supported by the Korean Science and Engineering Foundation and Ministry of Education.

${ }^{20}$ Supported by the World Laboratory.

${ }^{21}$ Now at California Institute of Technology, Pasadena, CA 91125, USA.

${ }^{22}$ Now at Dipartimento di Fisica, Università di Milano, Milano, Italy.

${ }^{23}$ Also at CERN, PPE Division, 1211 Geneva 23, Switzerland.

${ }^{24}$ Now at DESY, Hamburg, Germany.

${ }^{25}$ Now at TRIUMF, Vancouver, B.C., Canada.

${ }^{26}$ Also at Università di Napoli, Dipartimento di Scienze Fisiche, Napoli, Italy.

${ }^{27}$ On leave of absence from IHEP, Beijing, The People's Republic of China.

${ }^{28}$ Now at Harvard University, Cambridge, MA 02138, U.S.A.

${ }^{29}$ Supported by the US Department of Energy, grant DE-FG03-92ER40689.

${ }^{30}$ Visitor from University of Wisconsin, Madison, WI 53706, USA.

${ }^{31}$ Now at Università di Pavia, Pavia, Italy.

${ }^{32}$ Now at Max-Planck-Institut f. Kernphysik, Heidelberg, Germany.
} 


\section{Introduction}

The results from LEP have confirmed the standard electroweak model, and the production and properties of hadronic events are consistent with expectations based on QCD. A widely accepted premise of the Standard Model is that quarks are confined. However, confinement is not necessary in the Standard Model [1] and has not been proven theoretically in QCD. Rather, it is invoked to explain the experimental fact that no free quarks have been detected. Whether free quarks can be produced is still one of the open fundamental experimental questions. On the $\mathrm{Z}$ pole quarks are produced with high momenta mainly by the weak interaction and might become free without contradicting the numerous limits given by experiments at lower $e^{+} e^{-}$centre of mass energies [2, 3, 4, 5, 6, 7, 8, 9]. A number of searches for "new" particles have previously been carried out in ALEPH, and limits have been set on the production of new, heavy particles in the decay of the $Z$. These searches have assumed that heavy unusual particles would decay to known charged particles before reaching the tracking chambers, according to characteristic signatures. Neutral, long-lived, weakly-interacting particles have also been excluded [10,11, 12]. The present search is complementary to these, and sets limits on the production of long-lived charged particles with ionization losses not expected by standard mass and charge assignments.

This study concentrates on the search for new charged particles whose interaction length is comparable to or larger than that of the stable hadrons ${ }^{1}$ and which appear in pairs, usually accompanied by jets $\left(e^{+} e^{-} \rightarrow x \bar{x}+\right.$ hadrons). Single production of unusually ionizing particles is also studied within the kinematic limits, covering the case of singly produced long lived charged particles and the case where a conservation law is maintained by the production of a low mass partner, as in some extensions to the Standard Model .

The main tracking detector of ALEPH, the Time Projection Chamber (TPC), has been designed to measure the ionization loss of charged particles and has been used extensively in particle identification in ALEPH publications. It is also well suited for a search for charged tracks with ionization losses inconsistent with the expectation for known particles.

In Fig. 1 the expected ionization loss $(d E / d x)$ curves of known particles in the ALEPH TPC are shown, normalized to a minimum ionizing particle (MIP), as a function of the apparent momentum (momentum divided by the magnitude of the charge). These lines are drawn according to the empirical formula [13],

$$
\frac{d E}{d x}=\xi \frac{q^{2}}{\beta^{p}}\left(K+2 \ln (\beta \gamma)-\beta^{p}-\delta(\beta)\right),
$$

where $q$ is the charge in units of the electron charge, $\beta$ and $\gamma$ are the usual Lorentz variables, and $\xi, p, K$, and the polynomial $\delta(\beta)$ are obtained from the ALEPH data [14]. A scatter-plot of $d E / d x$ versus apparent momentum from part of the data sample is shown in Fig. 2.

The signature of a fractionally charged or heavy, long-lived charged particle would appear as an entry in Fig. 2 well outside the bands populated by the known particle species. In Fig. 1 the expected curves for fractionally charged particles $(q=1 / 3,2 / 3,4 / 3)$ of mass $5 \mathrm{GeV} / c^{2}$ are shown, indicating the sensitive regions for this analysis. The low ionization region $\mathbf{I}$ is bounded from above by a line drawn $4 \sigma$ below the mean ionization of the known $q=1$ particles, and from below by the detection efficiency of the detector. The high ionization region II is bounded from below by a line $4 \sigma$ above the mean ionization of the known $q=1$ particles, from the left by the ionization curve of a mass $1.2 \mathrm{GeV} / c^{2}, q=1$ particle, and from above by the limits imposed by the dynamic range of the TPC electronics. The boundaries are drawn for an average resolution. Copiously produced heavy new particles would form a band about a curve specific to their charge and mass.

\footnotetext{
${ }^{1}$ Assuming an interaction cross section of ten times that of a $5 \mathrm{GeV} / \mathrm{c}$ pion and the given amount of material before the TPC active volume, the sensitivity of this experiment extends to interaction lengths larger than about $0.12 \mathrm{~g} / \mathrm{cm}^{2}$.
} 


\section{The ALEPH Detector}

The ALEPH detector is described in detail elsewhere [15]. Only a brief description of the features relevant to this analysis is given here. Close to the beam pipe is the inner tracking chamber (ITC), with eight concentric drift chamber layers of active length $200 \mathrm{~cm}$, inner radius $12.8 \mathrm{~cm}$ and outer radius $28.8 \mathrm{~cm}$. The ITC is used in both the track trigger and the track reconstruction.

Surrounding the ITC is the large Time Projection Chamber (TPC), a cylindrical, threedimensional imaging drift chamber with 18 multiwire proportional chambers (sectors) at each end, with active length $2 \times 220 \mathrm{~cm}$, inner radius $31 \mathrm{~cm}$ and outer radius $180 \mathrm{~cm}$. It has an axial electric field, of $120 \mathrm{~V} / \mathrm{cm}$, parallel (or antiparallel) to the $1.5 \mathrm{~T}$ magnetic field and of opposite direction in the two halves, which are divided by a conducting membrane located in the $z=0$ plane. Electrons from ionization drift to the end plates where they are detected by wire chambers with cathode planes that are segmented into concentric rows of $3 \mathrm{~cm}$ long by $6.2 \mathrm{~mm}$ wide pads. The cathode-pad rows provide measurements of up to 21 space points for charged particles. Charged particles within $|\cos \theta|<0.96$ cross all 8 layers of the ITC and at least 4 cathode-pad rows of the TPC. In addition, up to 330 measurements of the ionization loss, $d E / d x$, for each charged particle are provided by the TPC sense wires. In hadronic events the $d E / d x$ resolution obtained is $4.4 \%$ for 330 ionization samples. The material before the TPC active volume corresponds to 0.065 radiation lengths and 0.015 interaction lengths.

The electromagnetic calorimeter (ECAL) is a highly segmented sandwich of wire chambers and lead plates, with a thickness of 22 radiation lengths and covering the polar angle region $|\cos \theta|<0.98$. The position and energy of the electromagnetic showers are measured using $3 \times 3 \mathrm{~cm}^{2}$ cathode pads connected internally to form projective towers. Each tower is read out in three stacks with depths corresponding to 4,9 , and 9 radiation lengths. For electromagnetic showers the energy resolution is measured to be $\Delta E / E=0.18 / \sqrt{E}(E$ in $\mathrm{GeV})$.

The ITC, TPC, and ECAL are enclosed in a superconducting solenoid which provides an axial magnetic field of $1.5 \mathrm{~T}$. The $120 \mathrm{~cm}$ thick return yoke of the magnet is instrumented with $1 \times 1 \mathrm{~cm}^{2}$ streamer tubes to form the hadron calorimeter (HCAL), covering the angular region $|\cos \theta|<0.99$.

The triggers for hadronic events have been described in detail in other publications [15, 16]. They depend on having a charged trigger from the ITC and a particle penetrating the HCAL in the same azimuthal region as the ITC signal or an ECAL energy deposition greater than $0.2 \mathrm{GeV}$ $(1.3 \mathrm{GeV}$ in 1989$)$ in a module in the same azimuthal region as the ITC signal. The overall trigger efficiency for hadronic Z decays is $100 \%$. The trigger efficiency for the present search is the reported $100 \%$ only when the ionization of at least one track in the event gives an ITC trigger. For the study of two body production of fractionally charged particles the efficiency is assumed to be zero when the expected energy loss in the ITC is less than that of a MIP.

\section{Event Selection}

ALEPH data from 1989 and 1990, with over 180000 hadronic and leptonic events corresponding to $8 \mathrm{pb}^{-1}$ of data, have been used in the present analysis. An event was accepted in the analysis if there was an ALEPH charged trigger, as described above, and all detectors were active. Runs with high backgrounds in the TPC were excluded. The event sample for this study included low multiplicity events which are usually classified as two photon or beam gas events.

Tracks were considered as candidates for the high ionization region II if they had at least 6 TPC and 4 ITC hits used for the helix fit, which effectively limits the polar angle to the range of $24.5^{\circ}$ to $155.5^{\circ}$. The $\chi^{2} /$ dof of the helix fit was required to be less than 4 . The $d E / d x$ measurement was required to come from at least 150 wires, which gives in the worst case a resolution of $5.5 \%$. In 
order to eliminate $\delta$-rays and nuclear fragments coming from secondary interactions in the beam pipe and the walls of the tracking detectors, a stringent cut was imposed on the minimum distance of the track helix to the main interaction vertex of the event, expressed in the plane perpendicular to the beam as $\left|d_{0}\right|<0.5 \mathrm{~cm}$, and parallel to the beam as $\left|z_{0}\right|<5.0 \mathrm{~cm}$. For the low ionization region looser track selection criteria were used, since the lower ionization energy loss may result in fewer and worse coordinate and fewer $d E / d x$ measurements in the tracking detectors. In this case charged tracks were required to have at least $4 \mathrm{TPC}$ hits used for the fit, and may have as few as zero ITC hits. The $\chi^{2} /$ dof of the helix fit in this case was required to be less than 9 . The $d E / d x$ measurement was required to come from at least 80 wires. The distance to the event vertex was required to satisfy $\left|d_{0}\right|<2.0 \mathrm{~cm}$ and $\left|z_{0}\right|<10 \mathrm{~cm}$.

In order to identify and reject tracks appearing as candidates in the low ionization region I due to a malfunction in the TPC readout electronics which affected $0.1 \%$ of the events, a check of the raw wire digitizations was made. Track candidates which had contributions from digitizers which in the same event gave internally inconsistent measurements, or track pulse height distributions inconsistent with a Landau distribution, were rejected.

Due to the finite two track resolution of the TPC, two same-sign tracks with momenta larger than $5 \mathrm{GeV} / \mathrm{c}$ and an opening angle less than two degrees often are not properly resolved [14]. In order to increase the rejection of such candidates, additional cuts were applied in the track selection and $d E / d x$ evaluation. Use of the independent pad pulses was made to reject tracks associated with overly broad pad clusters, and tighter cuts were applied in the association of wire pulses with tracks than were used in the standard reconstruction.

In order to exclude the background from deuteron and tritium particles, a band within $2.5 \sigma$ from the expected mean ionization of these particles has been excluded. All tracks which were rejected by this cut and were well separated from the electron band have been found to have positive charge. To exclude remaining beam gas or two photon events from the set of candidates, a cut on the charged energy imbalance along the $z$ direction was applied. An event was rejected as a beam gas event if

$$
\frac{\left|E_{+}-E_{-}\right|}{\left|E_{+}+E_{-}\right|}>0.95 \text { and } E_{\text {cal }}<50 \mathrm{GeV},
$$

where $E_{+}\left(E_{-}\right)$is the summed energy of charged tracks with $\cos \theta>0(\cos \theta<0)$ and $E_{\text {cal }}$ is the total calorimetric energy of the event. It has been checked with the Monte Carlo sample that this cut keeps all hadronic $Z$ events at the level of $99.99 \%$. For the 8 events that are removed by this cut, the candidate track has low momentum, positive charge, and $d E / d x$ less than $4 \sigma$ away from a nuclear band.

Finally, 9 candidates remain in region I and 35 candidates in region II (Fig. 3). A visual scan of these candidates confirms that they have no accompanying signature that would distinguish them from normal hadronic or leptonic $Z$ events other than the high or low ionization of the one track.

The backgrounds were extensively studied using a hadronic Monte Carlo event sample. All the qualitative features of the data are reproduced. A clear two track overlap band appears in region II. The boundaries of this band, shown in Fig. 3, are defined by the overlap of the expected ionization of pairs of particle types, excluding the combinations for which both tracks are electrons.

The candidate tracks in both regions have no striking signature in the calorimeters, and there are no unusual energy flow patterns in the events. An isolation angle cut of $20^{\circ}$ from the nearest neighbour with momentum larger than $1 \mathrm{GeV} / c$ rejects all of these candidates. Thus for specific models which would predict such isolation of the particles of interest, the number of candidates is zero without any further cuts.

The 9 candidate tracks in region I which pass the $4 \sigma$ cut designed to exclude the bulk of the 
background are all less than $5 \sigma$ away from a known particle ionization curve. All of these tracks have low momenta, corresponding to masses less than $5 \mathrm{GeV} / c^{2}$. The average charged hadron momentum in the data is $2.5 \mathrm{GeV} / c$, and the minimum of the ionization curves, as can be seen in Fig. 1 and Fig. 2, is very densely populated by low momentum particles. It is thus reasonable that the background which survives the $4 \sigma$ statistical cut is concentrated below $2.5 \mathrm{GeV} / \mathrm{c}$ momentum.

The 35 candidates in region II can be grouped as follows:

- Two tracks reconstructed as one due to the jet-like nature of the hadronic Z decays and the limits imposed by the detector granularity (i.e. cases where two same-charge tracks are close together in space and momentum). The energy deposition in the calorimeters is consistent with this interpretation in each case.

- Beam gas events that just pass the asymmetry and energy cuts. They have a positively charged track with momentum less than $1 \mathrm{GeV} / c$ which is lying less than $3.5 \sigma$ away from the deuteron ionization curve.

- Electrons (positrons) with multiple overlapping delta rays, resulting in ionization values which pass the $4 \sigma$ cut. Each is found to have an electron signature in the ECAL.

A cut which excludes a band of the sensitive region in the area of the double track ionization reduces to 3 the number of events in region II. In this case a track is rejected if its $d E / d x$ measurement is within $2.5 \sigma$ from the expected ionization of any combination of double tracks of known charged particles. The remaining three events in this region are two beam gas candidates, which fall just beyond the cuts applied in relation 2 and are less then $3.5 \sigma$ from the deuteron ionization band, and a candidate consistent with being an electron, as determined from the calorimetric signature. All three have momenta less than $1 \mathrm{GeV} / c$.

Thus in total there are 9 candidates in the low ionization region I, and 3 in the high ionization region II, all of which can be attributed to remaining known background, and which correspond to masses smaller than $5 \mathrm{GeV} / c^{2}$, as can be seen in Fig. 1. As the Monte Carlo simulation of the tails of the ionization distribution have large systematic errors, the limits in this low mass region would depend on the particular parameterization chosen for the background. In the next section the choice is made to report limits for mass regions higher than $5 \mathrm{GeV} / c^{2}$, where with the cuts applied there are zero observed candidates.

\section{Mass Limits}

The limit for the production of new charged particles from this search can be expressed in terms of the dimuon cross section by the ratio

$$
R_{x}=\frac{\sigma(x \bar{x})}{\sigma\left(\mu^{+} \mu^{-}\right)} \quad \text { or } \quad R_{x}=\frac{n}{\varepsilon \cdot n_{\mu^{+} \mu^{-}}} .
$$

The number of dimuon events for the luminosity of the data sample used in this search is 6388 . The only unknown in this ratio is the efficiency $\varepsilon$ for detecting these new particles.

The efficiency for detecting a particle with mass $m$ and charge $q$ in a hadronic event depends on the model for its production and can be expressed as

$$
\varepsilon \equiv \varepsilon(q, m)=\frac{\int_{p_{\min }}^{p_{\max }} \frac{d N}{d p} \varepsilon(p, q) d p}{\int_{p_{\min }}^{p_{\max }} \frac{d N}{d p} d p}
$$

with $p$ the momentum of the particle. Two extreme models have been used for the momentum distribution $d N / d p$ of the new particles. In Model I the momentum dependence is taken from a 
fit to the $x_{B j}$, the standard Bjorken variable, distribution of the hadrons in the ALEPH data [19]. In Model II the momentum distribution for the produced particles is given by

$$
E \cdot \frac{d N}{d p^{3}}=\text { Constant }
$$

and can be considered as a parameterization of the fragmentation function for the production of massive particles [18]. These two models cover the possible momentum phase space for the production of a new and unknown particle, and give an estimate of the dependence of the limits on the fragmentation function.

Monte Carlo events were used to calculate the efficiency $\varepsilon(p, q)$. It can be evaluated as a product of individual efficiencies

$$
\varepsilon(p, q)=\varepsilon_{c}(p) \cdot \varepsilon_{d}(p, q),
$$

assuming no correlation between them. The efficiency $\varepsilon_{c}(p)$ of the applied cuts, which defines the acceptance, was found from standard hadronic Monte Carlo events and ranges between 0.6 and 0.95. The efficiency $\varepsilon_{d}(p, q)$ that a track with charge $q$ and momentum $p$ within the acceptance would produce enough ionization to be detected in the TPC was found for fractionally charged particles in the low ionization region I by a special Monte Carlo simulation, with typical values of 0.6 for charge $q=2 / 3$ and 0.1 for charge $q=1 / 3$. The corresponding efficiency for $q=1$ particles and for fractionally charged particles in the high ionization region II is assumed to be $100 \%$, as is known to be the case for normal tracks. The efficiency for an exclusively produced pair of long lived quarks to give a charged ITC trigger is $100 \%$ for ionization equal to or larger than that of a MIP [15].

In the production of high mass particles the efficiency $\varepsilon_{c}$ is underestimated since the masses of all particles in the Monte Carlo are the standard particle masses. Pair production of heavy particles would leave little energy for large pion multiplicity. Therefore the tracks would be few and well separated, resulting in increased detection efficiency. Any efficiency numbers which take this effect into account would depend on detailed models of production and fragmentation, and therefore, no correction has been applied.

After the cuts described in Section 3 no candidates remain in the data for masses larger than $5 \mathrm{GeV} / c^{2}$. Limits are given using equation 3 with $n_{\mathrm{ev}}=2.3(90 \%$ C.L.). They are shown as a function of the mass of the produced particles in Fig. 4 for a momentum distribution produced according to Model I. The limit contours for the inclusive production ratio $R_{x}$ obtained from this analysis and from other $e^{+} e^{-}$experiments $[7,9]$ at lower energies are displayed in Fig. 5 for particles with $q=1 / 3,2 / 3,4 / 3$ and 1 , assuming the momentum distribution of Model II. It is seen that the present analysis extends the limits given by previous experiments in the mass range $15-70 \mathrm{GeV} / \mathrm{c}^{2}$. Table 1 shows the limits given by the present analysis for the exclusive reaction $\left(e^{+} e^{-} \rightarrow x \bar{x}\right)$. In this case, for a given mass of $x$ the momentum is uniquely determined, and discontinuities are introduced in the mass plots by the definition of the sensitive regions in Figure 1. The trigger efficiency for an event consisting of a single track with ionization less than that of a MIP cannot be easily estimated and is assumed to be zero. This reduces drastically the effective sensitive area for the exclusive production of $q=1 / 3$ and $q=2 / 3$ particles, and no limits are given in the present study for this case.

\section{Conclusion}

An analysis based on $d E / d x$ measurements in the ALEPH central tracking detector, the TPC, has been performed for all data collected during the 1989 and 1990 running periods of LEP. A total 


\begin{tabular}{||c|c|c||}
\hline charge & mass $\left(\mathrm{GeV} / c^{2}\right)$ & $R_{x}<$ \\
\hline \multirow{5}{*}{$q=4 / 3$} & $5.0-10.2$ & $3.6 \cdot 10^{-4}$ \\
& $16.5-26.0$ & $3.6 \cdot 10^{-4}$ \\
& $26.0-33.3$ & $6.9 \cdot 10^{-4}$ \\
& $33.3-38.6$ & $9.1 \cdot 10^{-4}$ \\
& $38.6-44.9$ & $1.1 \cdot 10^{-3}$ \\
\hline \multirow{4}{*}{$q=1$} & $34.4-39.6$ & $9.1 \cdot 10^{-4}$ \\
& $39.6-42.8$ & $1.10 \cdot 10^{-3}$ \\
& $42.8-44.9$ & $1.18 \cdot 10^{-3}$ \\
\hline
\end{tabular}

Table 1: The limits for exclusive two body channels. Production rates greater than the ones specified here are excluded at $90 \%$ C.L.

of more than two million tracks with $d E / d x$ measurements was used. No cuts have been imposed specific to a model of the production of the particles for which this search is made. Only cuts on the quality of the data have been applied. The candidate events have no distinctive signature besides the ionization of the candidate track. Exclusion of a band around the background expected from track overlap reduces the number of candidates to zero for masses above $5 \mathrm{GeV} / c^{2}$. The limits obtained for the production of fractional charges are lower than those previously reported by $e^{+} e^{-}$ collider experiments and extend the excluded mass range to $43 \mathrm{GeV} / c^{2}$. In addition, heavy longlived particles of charge $q=1$ are excluded up to masses of $70 \mathrm{GeV} / c^{2}$. Such production of stable new charged particles is ruled out at the level of $10^{-3}$ of the dimuon production cross section.

\section{Acknowledgements}

We thank our colleagues of the SL division for the good performance of the LEP accelerator. Thanks are also due to the many engineering and technical personnel at CERN and at the home institutes for their contributions toward the success of ALEPH. Those of us not from member states wish to thank CERN for its hospitality. 


\section{References}

[1] J. Ellis, Physica Scripta Vol 23, (1981) 328-338.

[2] W. Bartel et al. , Z. Phys. C 6 (1980) 295.

[3] J.M. Weiss et al., Phys. Lett. B101 (1981) 439.

[4] M.C. Ross et al., Phys. Lett. B118 (1982) 199.

[5] H. Ahaira et al. (TPC/PEP4 Coll.), Phys. Rev. Lett. 52 (1984) 2332.

[6] W. Guryn et al. (CNTR Coll.), Phys. Lett. B139 (1984) 313.

[7] H. Albrecht et al. (ARGUS Coll.), Phys. Lett. B156 (1985) 134.

[8] T. Bowcock et al. (CLEO Coll.), Phys. Rev. D40 (1990) 263.

[9] I. Adachi et al. (TRISTAN Coll.), Phys. Lett. B249 (1990) 336.

[10] D. Decamp et al. (ALEPH Coll.), Phys. Lett. B236 (1990) 511-522.

[11] D. Decamp et al. (ALEPH Coll.), Phys. Lett. B262 (1991) 139-147.

[12] D. Decamp et al. (ALEPH Coll.),Phys. Reports 216 (1992) 253-340

[13] H. Breuker, Nucl. Instr. Methods A260 (1987) 329.

[14] W. Atwood et al., Nucl. Inst. Methods A306 (1991) 446-458.

[15] D. Decamp et al. (ALEPH Coll.), Nucl. Instr. Methods A294 (1990) 121.

[16] D. Decamp et al. (ALEPH Coll.), Phys. Lett. B231 (1989) 519.

[17] D. Decamp et al. (ALEPH Coll.), Z. Phys. C 48 (1990) 365-391.

[18] M. Suzuki, Phys. Lett. B71 (1977) 139, J.D. Bjorken, Phys. Rev. D 17 (1978) 171.

[19] D. Decamp et al. (ALEPH Coll.), Properties of Hadronic Z Decays and Tests of QCD Generators, (Submitted to Zeit. Phys. C). 


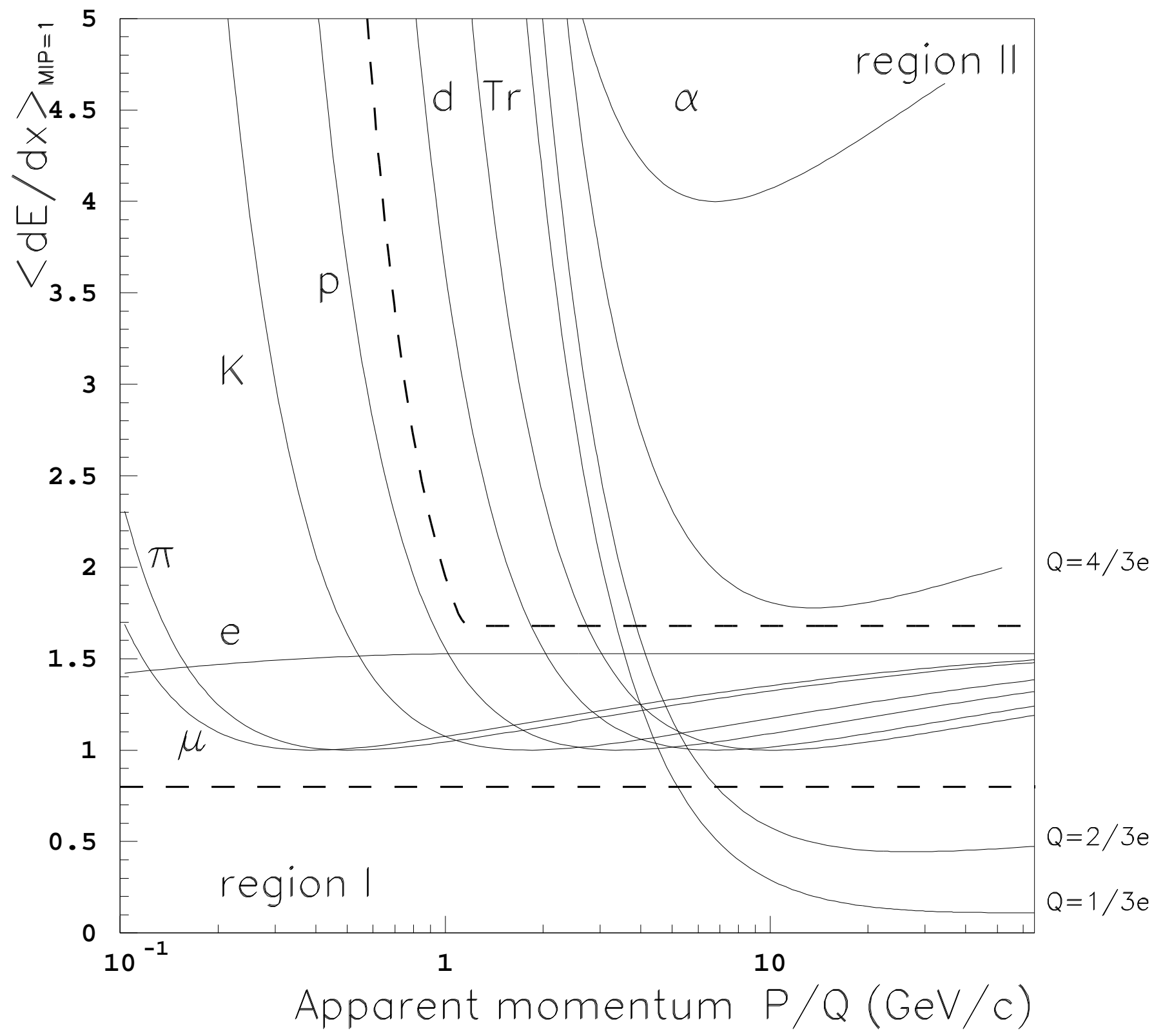

Figure 1: The expected ionization curves for different particle types. The curves for the fractional charges are for a mass $5 \mathrm{GeV} / c^{2}$ particle. The boundaries for the search regions are displayed in the case of an average resolution. 


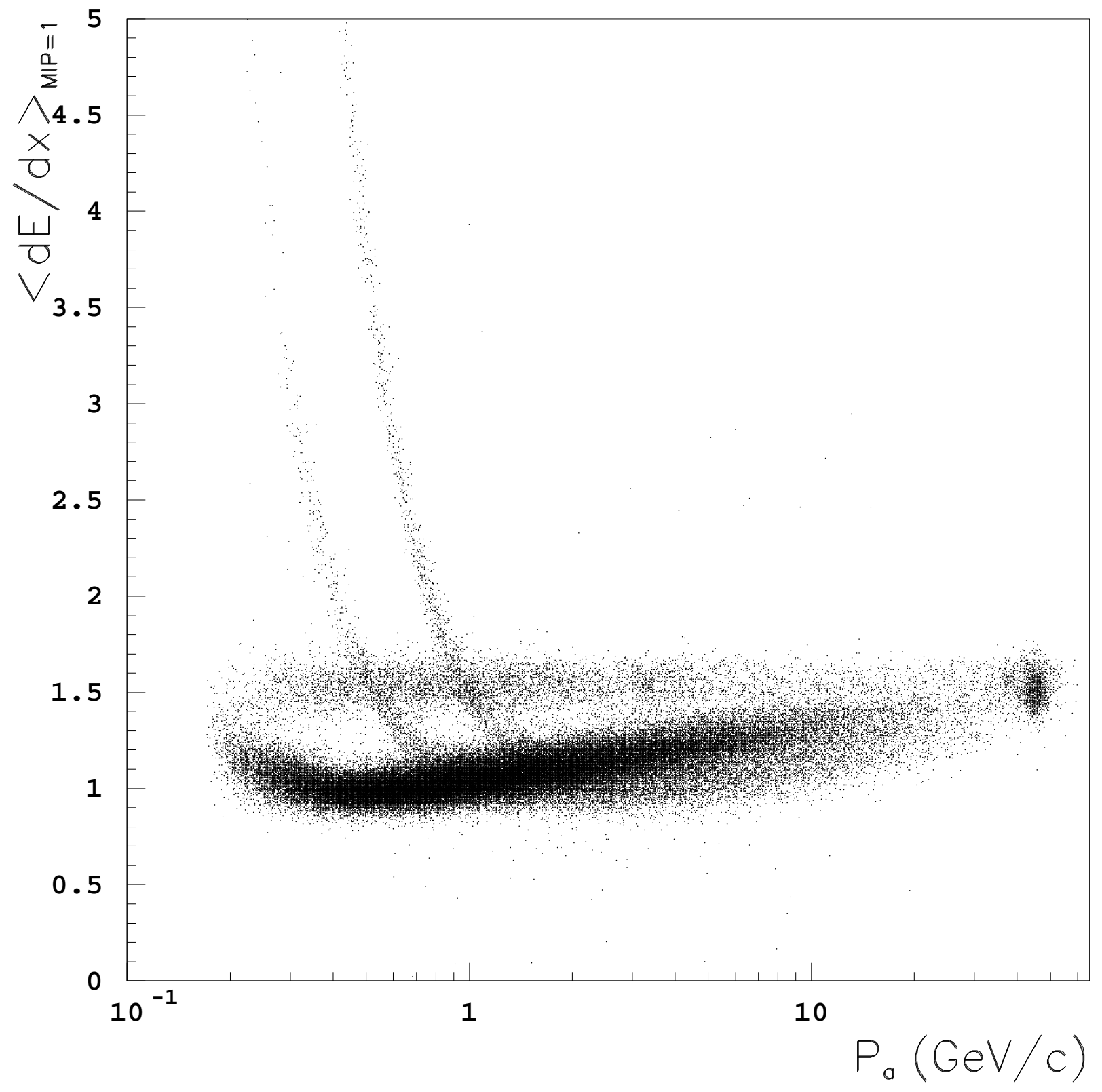

Figure 2: A scatter-plot of the truncated mean, normalized to unity for minimum ionizing particles, versus the apparent momentum is shown from a sample of events from 1989 and 1990 data which have been preselected for candidates. Only one in ten tracks outside the search regions is entered in the plot. 


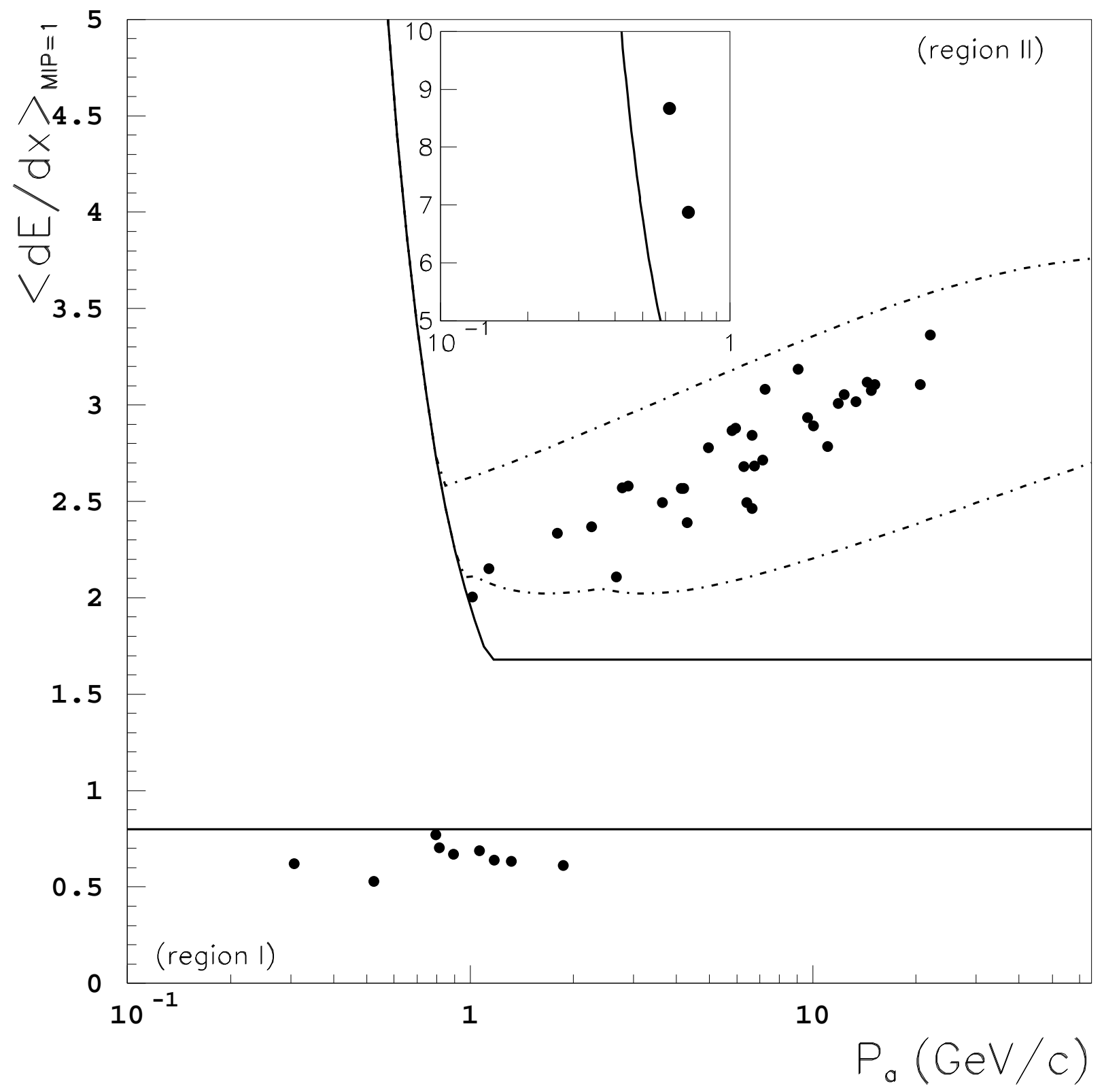

Figure 3: The final candidates in both regions I and II. The boundaries of the track overlap region are indicated by the dot-dash lines. All candidates for masses larger than $5 \mathrm{GeV} / c^{2}$ are within these boundaries. The low ionization candidates are attributed to a statistical excess below the $4 \sigma$ cut coming from the known $q=1$ particle distributions. There are two candidates shown in the insert. 

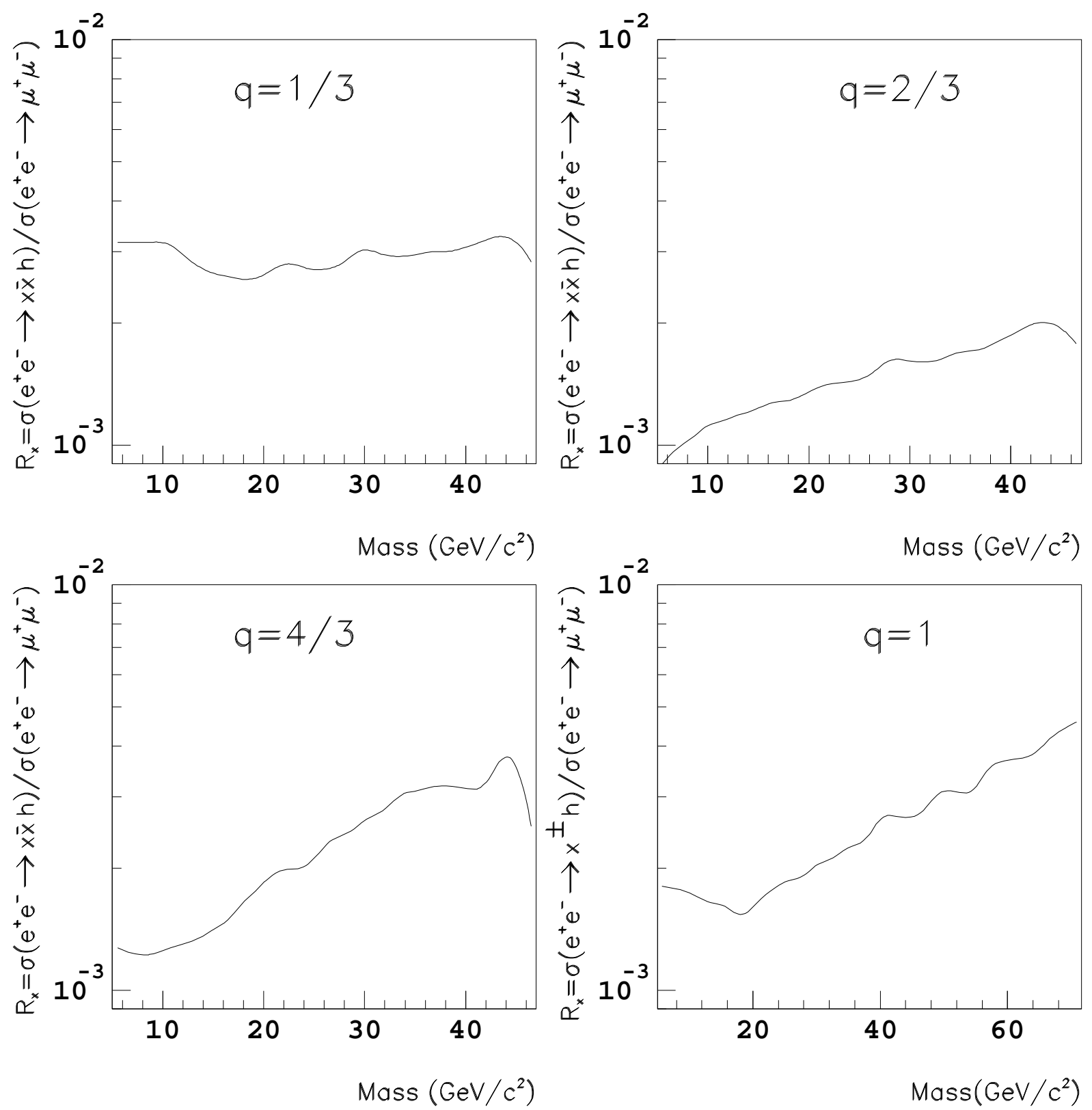

Figure 4: The 90\% C.L. limit for $R_{x}$ for the inclusive production of charged particles with unexpected mass and charge, assuming particles distributed in momentum according to Model I, which uses the ALEPH hadronic fragmentation function. The area above the curve is excluded. 

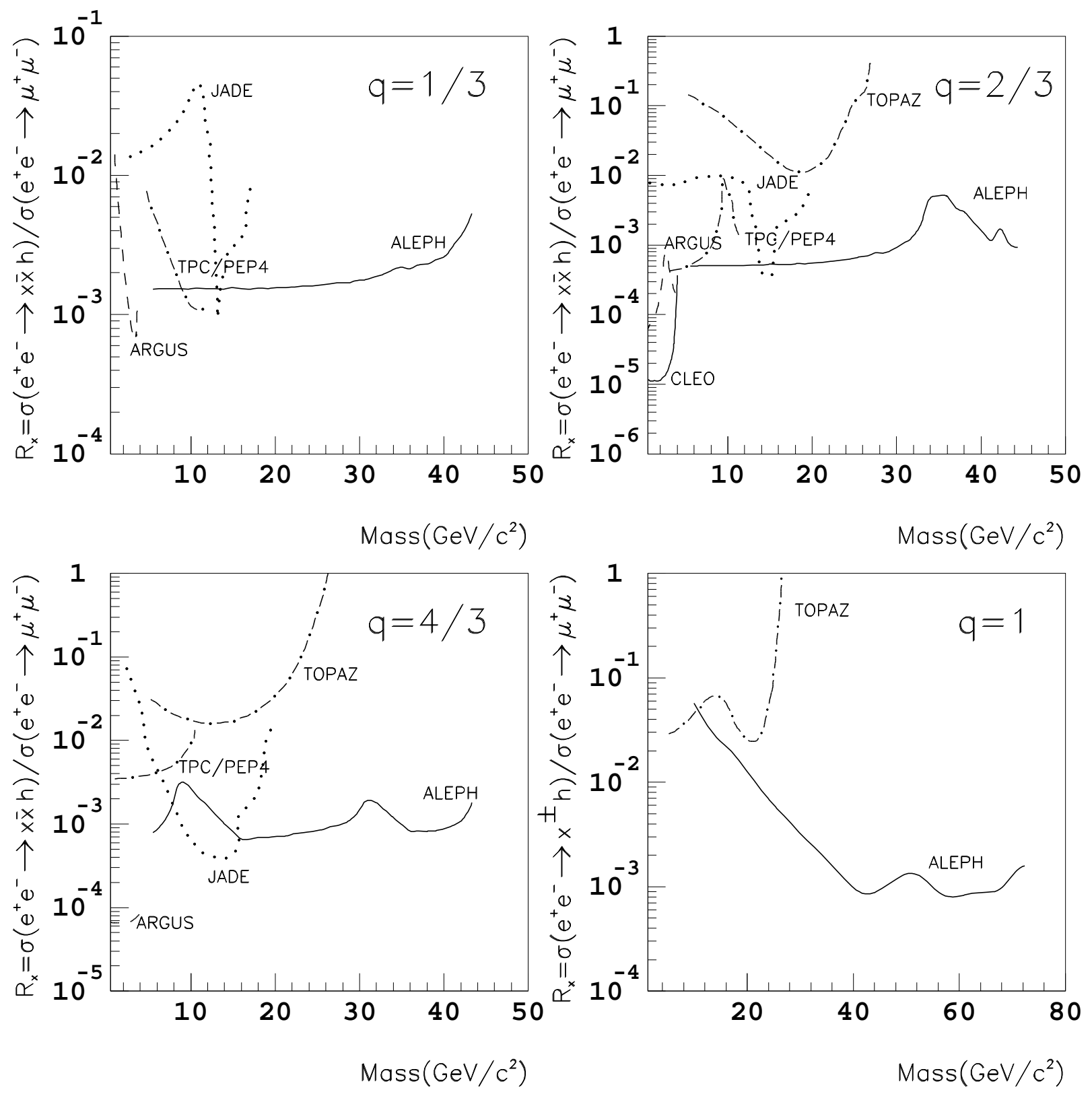

Figure 5: The 90\% C.L. limit for $R_{x}$ for the inclusive production of charged particles with unexpected mass and charge, assuming particles distributed in momentum according to Model II, from this and previous $e^{+} e^{-}$experiments. The area above the curve is excluded. 